champion support was available for all shifts for the first two weeks following this date. Standard Operating procedures for downtime were put in place and communicated.

Results A very successful implementation of a significant cultural change with no resistance from the team which has achieved the aims.

Conclusions Support and protected time was key, alongside involving the team and listening to their ideas and fears from the outset. By providing champions to work alongside staff they felt secure and protected in their learning

\section{P-243 DRIVING CHANGE IN HOSPICE CARE - REFLECTING ON THE SUCCESSES AND THE SCARS}

Jan Noble, Heather Richardson, Rob George, Penny Hansford, Ruth Sheridan, Jan Thirkettle. St Christopher's Hospice, London, UK

\subsection{6/bmjspcare-2017-hospice.268}

Aim A hospice in South East London has been engaged in a major programme of change to support its new strategic priorities. This presentation will provide an overview of the change management programme.

Methods The hospice recruited a senior manager to work as service development and transformation lead. Her focus was on change that would allow the hospice to reach more people using an increasingly finite resource of people and money.

Results Over the last two years the post-holder has been engaged in projects focused on:

- establishing the new Single Point of Contact

- designing and utilising a case management approach to guide the allocation of our resources (staff, skills and services)

- improving clinical decision making and related communications between professionals

- introducing a new electronic patient records system

- redesigning clinical administration services to better support clinical professionals.

Working closely with the different clinical directors she has adopted an approach broadly reflective of the PDSA cycle to design and implement change. The work has been overseen by a change board. Much of the change planned has been implemented relatively quickly and results are impressive. However, some resistance exists within the workforce, the impact on staff has been significant and further work is required to embed the changes and ensure new ways of working are sustained.

Conclusion Major change is possible within a hospice context, but it is challenging to implement. Dedicated and senior leadership is required to do this successfully; whilst careful attention to engaging staff in building a vision for something different prior to requiring them to change behaviours is a vital step.

\section{P-244 ASPIRING TO BE THE BEST WE CAN BE - A HOSPICE JOURNEY}

Elizabeth Arnold. Earl Mountbatten Hospice, Newport, UK

10.1136/bmjspcare-2017-hospice.269
The Care Quality Commission's (CQC) model of inspection changed in 2014 using a framework encompassing five key questions; is the service safe, effective, responsive, caring and well-led? The hospice planned for the inspection over many months, working with staff, volunteers and trustees to highlight areas of good practice and identify areas that required improving. The whole organisation engaged in workshops and interactive sessions together, fostering recognition that everyone has a responsibility to improve the quality of the services provided and the experiences of people who use our services.

Over 150 staff, trustees and volunteers came together periodically over the course of 18 months and informed us of their perceptions of the organisation's strengths and weaknesses. Through a process of engagement, listening and information gathering we formed a detailed picture of the hospice's position against the CQC's five domains. A detailed action plan was created together with files of 'evidence' for the CQC. The hospice received a rating of 'Outstanding'. It is without question that this achievement was wholly as a result of working together, collective leadership, trust and pride. A number of key themes emerged through this process which may be useful for other providers to replicate:

- What is the best that we can be? - developing a culture where people really believe in the values; these appeared essential to greater levels of engagement

- Leadership is about yourself - every person from 'ward to board' took personal responsibility and were supported to take the initiative and innovate

- Celebration and stories - the promotion of stories was seen as vital to emphasise the impact of what hospices do; one volunteer carried a photograph of an owl 'visiting' a patient to show the CQC should she be working when CQC visited!

- Our challenge is to be 'outstanding' across our entire organisation, every day and always.

\section{P-245 NOW WHERE IS THAT POLICY ON ...}

Paula Powell, Daniel Monnery, Susan Schofield, Charles Hyland, Debbie Jones. Willowbrook Hospice, Merseyside, UK

10.1136/bmispcare-2017-hospice.270

Most specialist palliative care units have clinical policies to support best practice. In addition multiple resources are available to clinical staff as books, clinical guidelines and apps. Our experience at Willowbrook Hospice was that staff would refer to guidelines and formats they were most familiar with even if newer versions were available. Equally comprehensive policies were not referred to because staff could not recall how to find them or when found the pertinent information was not easily accessible.

This represents a risk to patients and the organisation.

This poster describes the work that the medical and nursing teams carried out to develop hospice specific 'quick reference guidelines' for important policies and procedures that distilled the information into an accessible form, usually flow charts. Examples are - hypercalcaemia management, commencing ketamine, recognition and management of delirium and sepsis. 